\title{
Circuit
}

Musiques contemporaines

\section{Non! Mais acceptons que le jardin soit plus grand que vous ne le croyez!}

\section{Jean Piché}

Volume 1, numéro 2, 1990

Montréal musiques actuelles

URI : https://id.erudit.org/iderudit/902016ar

DOI : https://doi.org/10.7202/902016ar

Aller au sommaire du numéro

Éditeur(s)

Les Presses de l'Université de Montréal

ISSN

1183-1693 (imprimé)

1488-9692 (numérique)

Découvrir la revue

Citer cet article

Piché, J. (1990). Non! Mais acceptons que le jardin soit plus grand que vous ne le croyez! Circuit, 1(2), 51-54. https://doi.org/10.7202/902016ar d'utilisation que vous pouvez consulter en ligne.

https://apropos.erudit.org/fr/usagers/politique-dutilisation/ 


\section{Non! Mais acceptons que le jardin soit plus grand que vous ne le croyez! Jean Piché}

C'est à l'invitation de Jean-Jacques Nattiez que je viens livrer aux lecteurs de CIRCUIT quelques éléments de réponse aux propos tenus par lui et quelques collaborateurs sur le festival Montréal Musiques Actuelles/New Music America 1990. La programmation du festival, dont j'étais responsable, aura soulevé plusieurs questions de différents ordres. Si nous voulons que ce débat soit productif, plusieurs des points soulevés par JeanJacques Nattiez demandent une réponse non équivoque et plusieurs autres demandent des clarifications quant à nos intentions.

Définissons d'abord le paramètre essentiel de la question. Est-il légitime, en 1990 dans un festival de musiques actuelles, de faire coexister des pratiques musicales aussi diverses que le jazz d'avant-garde, la musique contemporaine de tradition classique, le rock expérimental, le multimédia, l'installation sonore, bref toutes les expressions qui peuvent potentiellement devenir les véhicules d'un geste créatif original ? Notre réponse: un OUI retentissant. Il est aberrant de reléguer cette acceptation du différent à une espèce d'idéologie-bonbon qui aurait pour principale caractéristique un manque total de discernement.

Premièrement, Montréal Musiques Actuelles était la onzième édition du festival américain New Music America. Son mandat même voulait que sa programmation soit concentrée sur la création musicale actuelle d'Amérique du Nord. Puisque l'événement se déroulait pour la première fois à l'extérieur des États-Unis, nous voulions refléter le plus fidèlement possible les différentes pratiques musicales actuelles canadiennes et américaines. La brochette était donc par nécessité bigarrée. Et on ne pourra pas nous reprocher d'avoir caché nos intentions puisque les grandes lignes devant guider la direction esthétique du festival avaient été longuement discutées avant sa tenue.

Le pétrissement et l'intégration des différents langages musicaux et des différentes pratiques musicales dans un même creuset auront touché (un 
peu à ma surprise d'ailleurs) un nerf bien vif chez les purs et durs de l'expression "classique». Aux exclamations outragées de mes collègues par ailleurs bien intentionnés, je ne peux que répondre: le ciel ne nous est pas encore tombé sur la tête! Pour provoquer de si hauts cris, notre piqûre était ou plus forte qu'on ne l'avait voulu, ou peut-être était-elle particulièrement bien ciblée! Force nous est d'examiner la blessure de plus près... Si on a trouvé une paille à notre œil, permettez-moi donc de retirer la poutre qui afflige le vôtre.

\section{Fascisme et démagogie?}

Il me semble un peu aberrant de lier une "démocratisation" de l'expression artistique au développement d'une tendance sociale fascisante, si bénigne soit-elle. Au contraire, ce qui m'apparaît hautement suspect, c'est bien une idéologie hiérarchisante qui veut non seulement faire prévaloir un langage sur tous les autres, mais en plus lui donner, en quasiexclusivité, les moyens de se faire entendre. De là à la domination d'un style officiel, que ce soit le réalisme socialiste ou l'interdit de séjour à la quinte et l'homorythmie, il n'y a qu'un pas. Dans cette optique, un fascisme en vaut un autre. Si la tolérance et la coexistence des idées ne sont plus une fonction vitale d'un humanisme éclairé, nous choisirons pour notre part de réorganiser, avec ou sans décibels, l'équilibre des hiérarchies qui ont permis cet état de choses. Bon goût et mauvais goût? Qui en sera l'arbitre et, surtout, comment définir dans la constellation des pratiques musicales contemporaines une hiérarchie qui soit à la fois juste et productive?

\section{Hiérarchie et vernaculaire}

La présence du vernaculaire dans le discours musical rehaussé a tou-jours été et reste pertinente. Les grandes formes historiques ont souvent été, si on fait exception de la dernière partie $d u x x^{e}$ siècle, intimement liées à l'expression populaire. Qui niera que Mozart et Beethoven étaient des musiciens proprement populaires! Rien, y compris la grande œuvre, n'existe en vase clos, si ce n'est les tenants d'un dialectisme étroit voulant formuler les règles d'un jeu où ils seront toujours gagnants. Le roi est nu? Et comment!

S'ensuit-il donc que toutes les musiques soient bonnes? Non. Et nous n'avons jamais donné cours à une telle affirmation. Ce que nous avons dit toutefois, nous le redisons sans équivoque: tous les genres de musiques 
peuvent trouver, dans les paramètres de leur expression propre, une manifestation de cette qualité d'excellence dont on fait état. Rejetterons-nous d'emblée la légitimité de tout un univers musical comme le jazz ou le rock? Miles Davis est-il un imbécile? Frank Zappa est-il un imposteur? La guitare électrique est-elle un instrument du diable? Et puis, pour bien fermer le cercle, la musique balinaise est-elle barbare parce que sans notation? Débat stérile et franchement réactionnaire. Si je peux reconnaître avec vous le talent exemplaire d'un Cherney ou d'un Montague (tous deux présents au festival), vous perdez à ne trouver qu'immondices chez Bruire, Scott Johnson ou Electric World.

Ce qui m'amène à cette malheureuse notion de "merde généralisée». Admettons tout de suite que merde il y eut et que merde il y aura. De là à en faire une tabula rasa, il y a une marge. S'il ne s'agissait que des choix spécifiques dont on parlait, on pourrait comprendre. Dans la mise en place d'un événement de la taille de Montréal Musiques Actuelles, il est normal que certaines prestations se soient avérées plus décevantes que d'autres. Je défie quiconque de produire une meilleure moyenne au bâton, y compris les grands festivals "sérieux» comme Musica à Strasbourg ou Huddersfield en Angleterre, où la merde est peut-être plus hiérarchisée mais demeure de la merde quand même.

C'est avec mainte délibération que nous avons choisi de considérer, d'un œil éclectique mais averti, la diversité des expressions existantes en Amérique. Pour reprendre l'analogie culinaire, si Bocuse ou Cagnat font de la grande cuisine, celui qui n'aura goûté que la cuisine française n'aura qu'une bien pâle idée des capacités gustatives du palais même le moins éduqué! La cuisine californienne ne se limite tout de même pas au McDonald.

\section{Économie politique}

Il y a tout un chapitre à écrire sur l'économie politique de la musique de la fin $d u x x^{e}$ siècle. La hiérarchie de valeurs si chère à l'élite doit-elle être construite sur un catéchisme de diktats et d'interdits? Puisque, aussi bien l'admettre tout de suite, la balance de l'argument réside dans la danse funèbre des subventions, qui aura droit à l'aide des deniers publics et qui décidera à qui ira cette aide? Serait-ce là le véritable mordant de notre piqûre?

Ah! oui, j'oubliais les jeunes. Et les vieux, et tous ceux entre les deux. Il est navrant de se voir reprocher d'essayer de présenter une musique intelligente, quoi qu'on en pense, à un auditoire généralisé qui n'a pas souvent la chance d'en entendre. Au risque de passer pour un démagogue, 
il est difficile de justifier une dépense énorme d'énergie et d'argent pour le plaisir raréfié d'une minorité éclairée. Qu'on considère celle-ci généreusement dans l'attribution des programmes, i'en suis, et nous l'avons fait. Mais de quel droit, public ou autre, peut-on proclamer un monopole sur l'excellence et les moyens avec lesquels elle s'exprimera? Ne vous en déplaise, le festival que nous avons organisé a donné une place plus qu'importante aux musiques dites "sérieuses" parce qu'elles sont et resteront un pivot important de l'expression contemporaine. Nous aurons au moins eu le courage, au nom de cet "idéalisme-gaga" qu'on nous reproche, de fouiller plus loin que les plates-bandes habituelles.

\section{La crise? Quelle crise?}

La musique "sérieuse» a perdu sa clé? Mais pourquoi s'entête-t-elle à la chercher seulement là où il y a de la lumière? C'est sans doute au niveau de l'ouverture des possibilités et de l'attitude que se retrouve la plus importante contribution "américaine» dans cette recherche, Cage et les post-cagiens en tête.

Et nous ne sommes pas seuls dans notre combat. Aux États-Unis, sans doute le pays à l'origine du débat académique contre vernaculaire, il aura été plus facile de passer rapidement à autre chose, la tradition (eh oui!) pesant moins lourdement sur les esprits créatifs. Même l'avant-garde musicale européenne (la Hollande en tête mais sans oublier les grognements de plus en plus impératifs en France et en Allemagne) mise aussi sur la déconstruction des styles comme solution possible à cette étonnante prémisse de la tradition musicale européenne qui veut que complexité égale intelligence et que simplicité égale démagogie. Qu'en penseraient un Rauschenberg (Américain), un Mondrian (Hollandais) ou un Malevitch (Russe)?! La crise, donc. Et de quelle crise parlions-nous?

L'histoire nous ferait-elle le coup encore une fois? La mise en demeure (l'expression est dure!) de l'hyper-complexité des écoles postsérielles et même postmodernes nous vient en direct de la musique minimale, mouvement américain s'il en fut. Ce renversement n'est pas sans rappeler le passage du baroque au classicisme au milieu du XVIII ${ }^{e}$ siècle alors que les fioritures ampoulées du haut baroque ont cédé la place à l'élégante simplicité d'un Haydn et d'un Mozart. Action-réaction. Si on ne réinvente pas l'histoire, on peut en examiner le processus. Le renversement des styles en musique comme dans les autres disciplines artistiques s'exerce souvent dans des circonstances conflictuelles et polémiques. Soit. Soyons prêts à en discuter et, $j^{\prime}$ en conviens tout de suite, on ne peut présumer du jugement de la mémoire. 